\title{
Cellules solaires en couches minces à base de silicium
}

\author{
Jean-Éric Bourée et Pere Roca i Cabarrocas
}

jean-eric.bouree@polytechnique.edu ; pere.roca@polytechnique.edu

Laboratoire de Physique des Interfaces et des Couches Minces, École Polytechnique, 91128 Palaiseau Cedex

Parallèlement à la filière

dominante du silicium

cristallin, et afin de

réduire les coûts de

manière substantielle,

les chercheurs

synthétisent, depuis

une trentaine d'années,

des cellules basées sur

le silicium désordonné

homogène (amorphe)

ou inhomogène

(nanocristallin, poly-

morphe). Dans ces

structures, déposées

à basse température

sur des substrats peu

chers (verre, plastique),

I'hydrogène joue un

rôle fondamental.

En combinant

les différents alliages

Si-Ge ou Si-C, on est

capable de réaliser des

cellules à multijonctions,

répondant de mieux

en mieux au spectre

solaire et devenant plus

stables en fonction du

temps.
En 2005 le silicium monocristallin (c-Si) et le silicium polycristallin, sous forme de plaquettes (wafers) ou de rubans, représentaient plus de $93 \%$ de la production mondiale de cellules solaires, estimée à 1,8 gigawatt-crête $\left(\mathrm{GW}_{\mathrm{c}}\right)$. Le reste concernait les modules en couches minces (d'épaisseur inférieure au micron) à base de silicium amorphe (4,7 \%), de CdTe ou de $\mathrm{CuInSe}_{2}$. Étant donné que les couches minces sont déposées sur des substrats bon marché comme le verre, l'acier inoxydable ou des feuilles de polymère, et que le coût des plaquettes de c-Si reste élevé (ce qui amène à un prix des modules supérieur à 2 euros $/ \mathrm{W}_{\mathrm{c}}$ ), il paraît inéluctable que le marché photovoltaïque des couches minces (dont l'augmentation annuelle est estimée à $60 \%$ contre $40 \%$ au marché photovoltaïque global) dépassera les $10 \%$ de la production mondiale d'ici cinq ans environ.

Dans ce contexte, la recherche photovoltaïque basée sur les couches minces de silicium amorphe doit se focaliser sur deux objectifs : i) réaliser des cellules à monojonction ayant un rendement de conversion stabilisé avoisinant $10 \%$ et, ii) à plus long terme, réaliser des cellules plus sophistiquées (à double ou triple jonction) à base d'alliages silicium-germanium ou autre, ayant un rendement stabilisé de l'ordre de $15 \%$.Après un rapide survol de l'évolution des cellules en couches minces de silicium amorphe à partir de la fin des années 70 , nous développerons ces deux aspects, en nous basant sur les résultats issus des laboratoires, et conclurons que le fort potentiel de cette filière justifie pleinement ces objectifs.

\section{Les dépôts par voie plasma à basse température}

L'aventure des cellules solaires à base de couches minces de silicium a réellement débuté en 1975 lorsque, contrairement aux prévisions théoriques de Mott, deux chercheurs britanniques, Spear et Le Comber, ont montré qu'il était possible de contrôler le dopage du silicium amorphe produit par la dissociation du silane $\left(\mathrm{SiH}_{4}\right)$ par décharge luminescente [1]. De fait, Spear et Le Comber avaient introduit de l'hydrogène dans la matrice silicium (avec une concentration atomique voisine de $10 \%$ ), ce qui n'était pas le cas lorsque les films de silicium amorphe étaient obtenus par pulvérisation cathodique ou dépôt sous vide. Comme indiqué dans l'encadré (p. 15), l'hydrogène introduit dans le matériau a une influence déterminante sur la densité de défauts inhérente au silicium amorphe.

Depuis les années 70, la technique adoptée industriellement et dans les laboratoires pour la synthèse de couches de silicium amorphe hydrogéné est la méthode dite PECVD, de dépôt chimique en phase vapeur assisté par plasma radiofréquence $(13,56 \mathrm{MHz})$ [2]. La figure 1 présente un schéma de réacteur PECVD et quelques réactions se produisant à la surface du dépôt [3]. Les gaz sources sont le

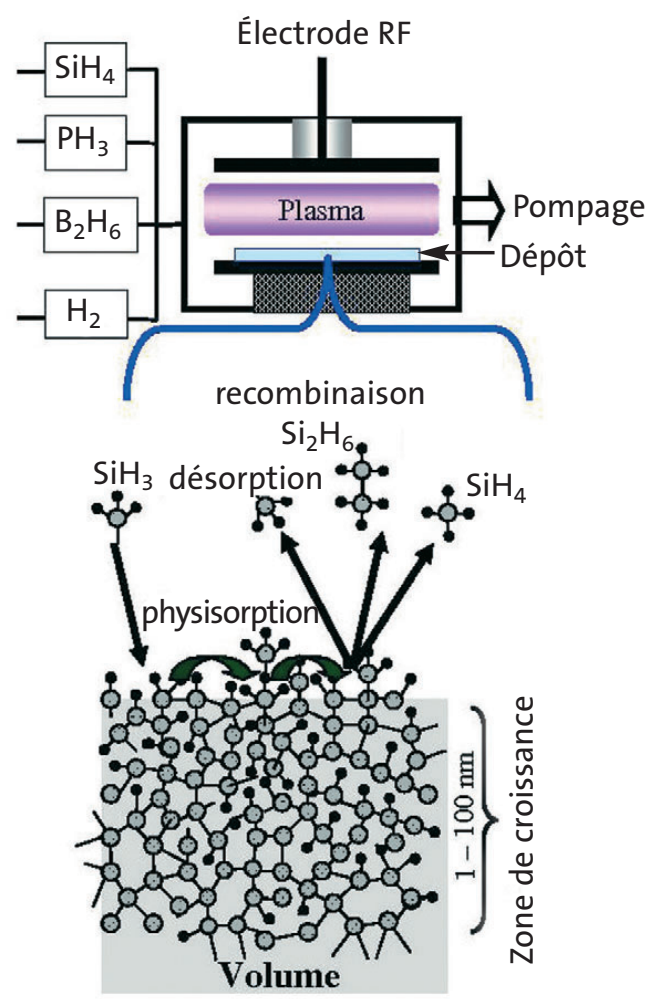

Figure 1. Dépôt de couches minces de silicium amorphe par décomposition chimique des gaz réactifs dans un plasma de décharge à basse température (on a représenté la surface de a-Si:H terminée par des liaisons $\mathrm{Si}-\mathrm{H}$ ). Les produits de dissociation des gaz précurseurs " condensent " sur le substrat, formant une couche mince. L'interface plasma/solide, appelée "zone de croissance ", implique des réactions contrôlées par la température du substrat et par l'énergie apportée par les ions et par l'hydrogène atomique (recuit chimique) [3]. 
silane, l'hydrogène et, pour les gaz dopants, le diborane $\left(\mathrm{B}_{2} \mathrm{H}_{6}\right)$ et la phosphine $\left(\mathrm{PH}_{3}\right)$. Dans le plasma, les collisions inélastiques entre les électrons de haute énergie (accélérés par le champ électrique RF) et le silane génèrent des radicaux, des ions et des espèces excitées. Les espèces réactives produites dans le plasma vont se condenser sur le substrat pour former (atome après atome) une couche mince, en général désordonnée. On peut ainsi déposer des couches sur de grandes surfaces (pouvant atteindre $4 \mathrm{~m}^{2}$ ), à (relativement) basse température (typiquement entre $100{ }^{\circ} \mathrm{C}$ et $300{ }^{\circ} \mathrm{C}$ ), donc sur des substrats peu chers.

Sur la base du silicium amorphe hydrogéné (a-Si:H), de nombreux développements se sont succédés, liés à la flexibilité du procédé de dépôt. L'ajout de germane $\left(\mathrm{GeH}_{4}\right)$ ou de méthane au silane dans la phase gazeuse a permis d'obtenir des alliages $a-\mathrm{Si}_{1-\mathrm{x}} \mathrm{Ge}_{\mathrm{x}}: \mathrm{H}$ ou a-Si $\mathrm{i}_{1-\mathrm{x}} \mathrm{C}_{\mathrm{x}}: \mathrm{H}$ et donc de synthétiser des matériaux ayant des largeurs de bande interdite (gaps) différentes, situées entre 1 et $2,2 \mathrm{eV}$ [6]. On a pu ainsi disposer d'une large palette de matériaux pour la réalisation de cellules multispectrales (voir encadré, p. 21). D'autre part, le fait d'utiliser un grand débit d'hydrogène moléculaire par rapport au débit de silane a permis, en cours de croissance, de générer suffisamment de radicaux d'hydrogène atomique, capables de graver plus efficacement le silicium amorphe que le silicium cristallin. Cet effet, combiné à l'apport d'énergie associé (recuit chimique), a conduit à la synthèse à basse température de couches nanocristallines de silicium. Ce silicium nc-Si:H (de taille de grains voisine de $30 \mathrm{~nm}$ ), est un matériau présentant un désordre inhomogène, caractérisé par des propriétés moyennes variant d'une région à une autre à l'intérieur du solide, et dont la mobilité électronique et la stabilité sont nettement améliorées par rapport à son analogue amorphe.

D'autres techniques de dépôt CVD ont vu le jour, dans le but d'augmenter la vitesse de dépôt ou de diminuer la densité des défauts. Parmi elles, citons la CVD à plasma micro-ondes, correspondant à un plasma de haute densité, ou la CVD assistée par filament chaud (HWCVD) qui engendre essentiellement des radicaux.

En parallèle, les études basées sur la CVD à plasma RF et concernant l'augmentation de la vitesse de dépôt ont conduit à s'intéresser à la formation des poudres dans les plasmas de silane. Ce phénomène, initialement perçu comme une nuisance, a ouvert la voie à une nouvelle gamme de matériaux. En effet, il a été montré que dans certaines conditions de la décharge, des nanocristaux de silicium étaient produits dans le plasma. Dès lors, nous avons essayé d'incorporer des nanocristaux dans la

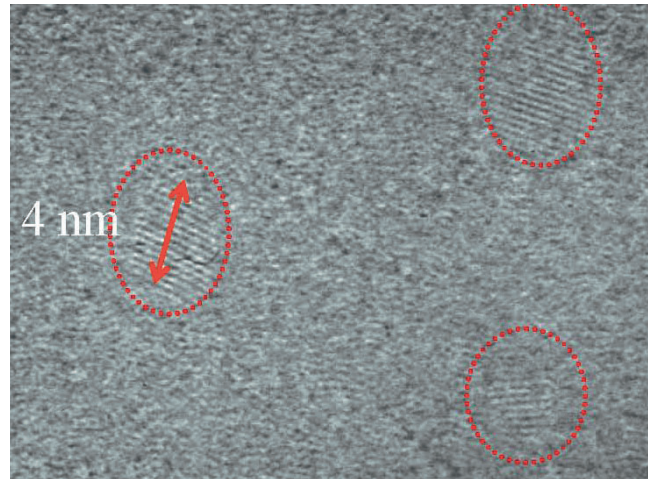

Figure 2. Images de microscopie électronique par transmission (TEM) en haute résolution: a) d'une couche de silicium polymorphe, où on distingue les nanocristaux de silicium dans la matrice amorphe ; b) d'une couche de silicium microcristallin épitaxiée sur GaAs et dont la croissance est obtenue à partir de nanocristaux formés dans le plasma. Le pointillé blanc indique l'interface GaAs/Si épitaxié et la ligne pointillée rouge montre que les plans cristallins de la couche de Si épitaxié suivent ceux du substrat GaAs.

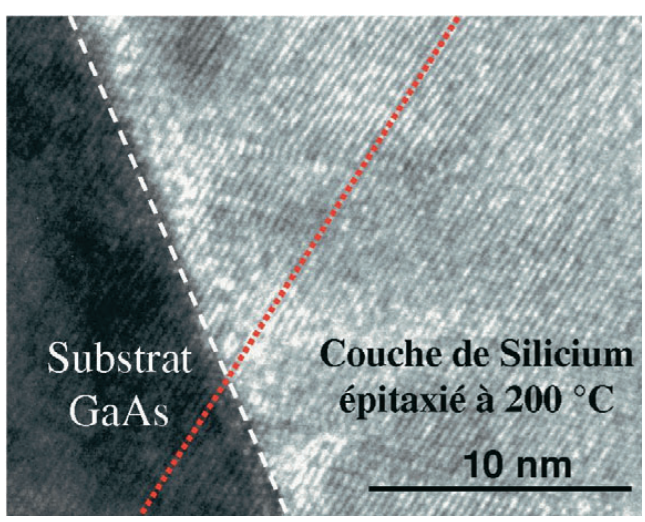

couche en cours de croissance. Le matériau ainsi obtenu, que nous avons appelé silicium polymorphe (pm-Si:H), est constitué de quelques nanocristaux de silicium dispersés dans une matrice amorphe (fig. 2a). Le pm-Si:H est un second exemple de matériau présentant un désordre inhomogène et peut être considéré comme du a-Si:H relaxé. On observe que ce matériau a une meilleure stabilité que a-Si:H, ce qui conduit à des cellules ayant un rendement proche de $10 \%$. Le pm-Si:H n'a pas encore livré tous ses secrets.

Les recherches sur la synthèse des nanocristaux dans un plasma à basse température $\left(200^{\circ} \mathrm{C}\right)$ se poursuivent, afin notamment d'élaborer des couches minces de silicium polycristallin à petits grains (de taille $>300 \mathrm{~nm}$ ). Nous avons récemment synthétisé une telle couche sur verre et aussi une couche épitaxiée sur GaAs monocristallin (fig. 2b).

La palette des matériaux synthétisés par plasma ne fait que s'enrichir et devrait faciliter l'éclosion de structures diverses réalisées en couches minces pour la production de cellules photovoltaïques à bas coût.

\section{Réalisation de cellules et modules}

Commençons par expliquer pourquoi une cellule en silicium amorphe hydrogéné, de gap voisin de 1,8 eV, a nécessairement une structure de type $\mathrm{p}-\mathrm{i}-\mathrm{n}$ ou $\mathrm{n}-\mathrm{i}-\mathrm{p}$ (i désignant une couche intrinsèque), et non pas une structure de type p-n ou n-p comme dans le cas des cellules au silicium cristallin (voir l'article de S. Martinuzzi 


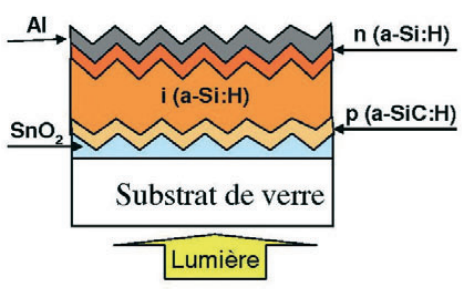

Figure 3. Schéma de base d'une cellule $p$-i-n en silicium amorphe hydrogéné. Dans le cas des cellules multispectrales, cette structure de base est répétée deux ou trois fois en jouant sur le gap de la couche intrinsèque. Ce dernier peut varier entre celui du a-Si:H (1,8 eV) et celui du silicium microcristallin (1,1 eV), en passant par celui des alliages a-SiGe:H (1,7-1,2 eV). On remarquera la structuration périodique des interfaces, permettant un meilleur piégeage optique.

(1) En silicium cristallin, on réalise des jonctions $\mathrm{p}-\mathrm{i}-\mathrm{n}$ au lieu de $\mathrm{p}-\mathrm{n}$ pour la détection de particules. Mais, pour les réaliser il faut un matériau de très haute pureté, donc de coût très élevé, ce qui interdit cette structure pour le photovoltaïque. dans Reflets de la physique $\mathrm{n}^{\circ}$ 5, p. 8). En effet, si l'on considère une structure $\mathrm{p}-\mathrm{n}$ amorphe, la densité de centres recombinants dus aux atomes d'impuretés et aux défauts, est tellement élevée que la durée de vie des photoporteurs devient très faible, de sorte qu'ils se recombinent avant de se séparer. Au contraire, en intercalant entre les couches $\mathrm{p}$ et $\mathrm{n}$ une couche non dopée (intrinsèque) avec une faible densité d'impuretés, la zone de charge d'espace s'étend largement dans la couche i et permet de collecter les porteurs par le champ électrique, de façon plus efficace que par diffusion(1).

La figure 3 montre le schéma d'une telle structure : sur un substrat en verre, recouvert d'une couche d'un oxyde transparent conducteur $\left(\mathrm{SnO}_{2}, \mathrm{ZnO}\right)$, on dépose successivement une couche dopée $\mathrm{p}$ d'une dizaine de $\mathrm{nm}$, une couche intrinsèque i ne dépassant pas $300 \mathrm{~nm}$ et une couche dopée n de $15 \mathrm{~nm}$. Le rôle des couches $\mathrm{p}$ et $\mathrm{n}$ est de créer le champ électrique qui permettra la collecte des porteurs photogénérés dans la couche i. On dépose ensuite par évaporation thermique ou par pulvérisation cathodique une couche métallique (Al, Ag...), qui sert de contact électrique vis-à-vis de la couche $\mathrm{n}$. Les améliorations successives de cette structure de base ont conduit à des cellules ayant un rendement proche de $10 \%$. On peut en citer quelques exemples : piégeage optique grâce à une couche $\mathrm{SnO}_{2}$ rugueuse (fig. 3), couche dopée $\mathrm{p}$ en alliage a-SiC:H (grand gap) pour réduire l'absorption dans la couche fenêtre qui ne contribue pas à l'effet photovoltaique, amélioration de la qualité de la couche intrinsèque (dépôt de pm-Si:H ou de nc-Si:H), réflecteur arrière pour maximiser le photocourant.

Un inconvénient majeur associé à l'utilisation du silicium amorphe hydrogéné pour les cellules solaires réside dans la dégradation du rendement de conversion sous l'effet de la lumière, qui induit des liaisons pendantes métastables pouvant piéger les porteurs. Si on ne peut supprimer cette dégradation, du moins peut-on la réduire, soit en modifiant le désordre local (emploi de nc-Si:H ou pm-Si:H ayant un désordre inhomogène), soit en diminuant l'épaisseur de la couche intrinsèque, qui doit rester inférieure à la longueur de diffusion des porteurs (voir Reflets $\mathrm{n}^{\circ 5}$, p. 9)

La structure $\mathrm{p}-\mathrm{i}-\mathrm{n}$ de la figure 3 est à la base du developpement industriel de la filière couches minces silicium [7]. Une manière d'améliorer efficacement le rendement et la stabilité des cellules en couches minces consiste à réaliser des cellules multijonctions empilées, en s'inspirant des hétérostructures mises au point pour les cellules à haut rendement basées sur les matériaux III-V (voir encadré, p. 21).
Logiquement, les premières cellules tandem (à double jonction) ont été basées sur une cellule avant de type a-Si:H (grand gap) et une cellule arrière de type a-Si $i_{1-x} \mathrm{Ge}_{\mathrm{x}}: \mathrm{H}$ ou de type nc-Si:H (petit gap), en utilisant le verre pour substrat. Un rendement initial de $13 \%$ a été mesuré sur une cellule a-Si:H/nc-Si:H, de surface $161 \mathrm{~cm}^{2}$. En 2006, Kaneka Corporation a produit $29 \mathrm{MW}_{\mathrm{c}}$ de modules sur la base de simples jonctions (rendement moyen $8 \%$ ) et de doubles jonctions (rendement moyen 10,3\%). Le temps de restitution en énergie pour ces modules est évalué à 1,6 ans et leur coût de production est estimé à 1,3 euros $/ \mathrm{W}_{\mathrm{c}}$. D'autre part, des cellules à triple jonction, de structure a-Si:H/a-Si $i_{1-\mathrm{x}} \mathrm{Ge}_{\mathrm{x}}: \mathrm{H} /$ $\mathrm{a}-\mathrm{Si}_{1-\mathrm{y}} \mathrm{Ge}_{\mathrm{y}}: \mathrm{H}$, déposées sur une plaque en acier inoxydable flexible de petite surface $\left(1 \mathrm{~cm}^{2}\right)$ ont montré un rendement initial de 14,6\%. Sur cette base, United Solar Ovonic a produit en 2006 $28 \mathrm{MW}_{\mathrm{c}}$ de modules avec un rendement moyen de $8 \%$. Bien entendu, toutes ces structures sophistiquées exigent la maitrise de la qualité des dépôts, quelle que soit la technique utilisée. Ce n'est que dans une seconde phase que l'accent est mis sur l'augmentation de la vitesse des dépôts, afin d'abaisser encore plus les coûts.

\section{Conclusion}

La recherche dans le domaine des couches minces à base de silicium pour le photovoltaïque, fait appel à une grande interdisciplinarité. En effet, plusieurs domaines méritent d'être largement développés, au niveau fondamental aussi bien que technologique : i) la physique des matériaux désordonnés inhomogènes (silicium polymorphe ou nanocristallin) qui n'est pas bien comprise ; ii) la physico-chimie des plasmas, dont les possibilités pour la synthèse de nouveaux matériaux réserve encore des surprises ; iii) la maitrise des interfaces (problèmes de passivation) qui jouent un rôle dominant dans la réalisation des dispositifs. Il est légitime de penser que les structures qui permettront d'atteindre, voire même de dépasser, $15 \%$ de rendement au niveau industriel, feront appel à des cellules hybrides impliquant le silicium polymorphe et le silicium nanocristallin.

En 2006, la production de la filière couches minces silicium a dépassé $120 \mathrm{MW}_{\mathrm{c}}$, alors que les productions des autres filières couches minces atteignaient respectivement $8 \mathrm{MW}_{\mathrm{c}}$ (CIGS) et $55 \mathrm{MW}_{\mathrm{c}}$ (CdTe). Au cours des prochaines années, la concurrence risque d'être rude entre la filière silicium et la filière CIGS, qui montera très rapidement en puissance. À cet égard, l'apport des équipementiers capables d'améliorer les techniques de dépôt existantes sur des grandes surfaces, ou capables d'en imaginer de nouvelles, sera déterminant. 


\section{Concepts associés aux solides désordonnés amorphes}

Les solides amorphes sont désordonnés topologiquement par nature, ne présentant aucun ordre à longue distance, mais ayant des propriétés homogènes à l'échelle macroscopique (sur un très grand nombre d'atomes). En d'autres termes, ils présentent un désordre homogène. Zachariasen a défini pour la silice vitreuse un modèle de réseau aléatoire continu, dans lequel chaque atome est entouré d'un nombre de voisins bien défini (modèle repris plus tard par Mott pour le silicium amorphe) : il existe un degré élevé d'ordre atomique à courte distance, lié à la nature de la liaison chimique remarquablement invariante à travers l'ensemble du solide. Au-delà des quatre premiers voisins, les atomes sont distribués de manière presque aléatoire.

Plus spécifiquement dans le modèle du silicium amorphe, chaque atome de silicium conserve le caractère tétravalent qu'il a dans le silicium cristallin (fig. A-a). Le principal défaut structural correspond à un atome entouré de trois voisins seulement, donc offrant un électron non apparié, ce qu'on appelle une liaison pendante (fig. A-b)

Dans l'approximation de Born-Oppenheimer, le mouvement des ions de cœur est séparé de celui des électrons de valence. Quand on considère l'état des électrons de valence dans le solide, les positions des ions sont de simples paramètres et un électron voit un potentiel effectif résultant des ions de cœur et des autres électrons de valence. Le désordre génère des potentiels effectifs fluctuant de manière aléatoire autour d'une valeur moyenne pour les électrons, ce qu a pour effet de localiser les fonctions d'ondes électroniques (le paquet d'ondes associé aux électrons est limité à des tailles inférieures au nm, comme cela a été démontré dans le cas du silicium poreux amorphe)

Ce modèle, développé par Anderson [4], puis affiné par Mott [5] dans les solides amorphes, conduit à des états électroniques localisés près des bords de bandes de valence et de conduction. La largeur de la "queue de bande" d'états localisés associée au désordre du matériau ne dépasse guère 0,5 eV, ainsi qu'il est montré sur la figure B présentant un schéma de la densité d'états électroniques du silicium amorphe. Dans ce schéma, les énergies $E_{v}$ et $E_{c}$ représentent les énergies seuil entre les états délocalisés (étendus à travers le solide) et les états localisés, et sont appelées seuils de mobilité côté bande de valence et côté bande de conduction respectivement. Les états localisés près des bords de bande proviennent de la distribution statis tique des angles de liaison et des distances interatomiques propres aux solides amorphes, alors que les états dans la bande interdite proviennent des défauts (essentiellement les liaisons pendantes) et dans une moindre mesure des impuretés (comme dans les solides cristallins). Entre les seuils $E_{v}$ et $E_{c}$, la conduction élec tronique se fait par sauts thermiquement activés entre états localisés, d'où une mobilité des porteurs très faible. Ceci correspond, sur la figure B, à ce qu'on appelle la bande d'énergie interdite pour la mobilité ou " gap de mobilité ».

Les centres de recombinaison électron-trou introduits dans le gap, dus à la présence des défauts, sont d'autant plus efficaces qu'ils sont proches du niveau intrinsèque. L'hydrogène introduit durant le dépôt a pour effet de saturer les liaisons chimiques insatisfaites (fig. A-c) et donc de réduire drastiquement la densité d'états localisés (fig. B), permettant ainsi le dopage des couches amorphes. C'est précisément ce qu'ont constaté Spear et Le Comber [1]

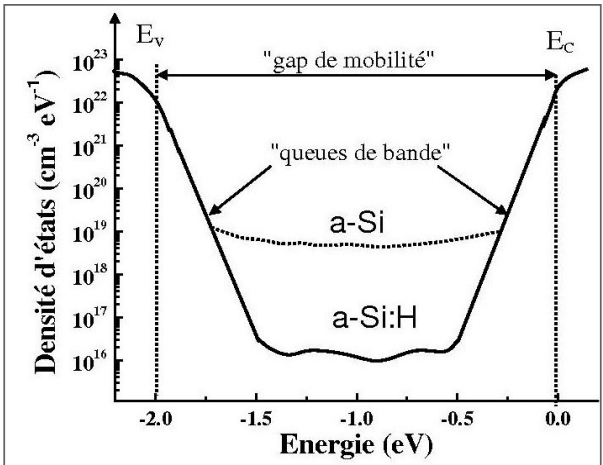

Figure B. Schéma montrant la densité d'états électroniques dans le silicium amorphe et le silicium amorphe hydrogéné.

On vient de le mentionner, la mobilité électronique dans le silicium amorphe est très faible, voisine de $1 \mathrm{~cm}^{2} / \mathrm{V} . \mathrm{s}$, alors qu'elle est de l'ordre de $1000 \mathrm{~cm}^{2} /$ V.s dans le silicium cristallin. Le libre parcours moyen des charges est donc aussi très faible (inférieur à $1 \mu \mathrm{m}$ ). En contrepartie, les défauts structuraux associés au matériau amorphe induisent une augmentation de l'absorption optique, qui est 5 à 10 fois supérieure à celle du c-Si dans la partie visible du spectre solaire (voir fig. 2, p. 7 de Reflets de la physique $\mathrm{n}^{\circ} 5$ ). Cette forte absorption de la lumière permet la photogénération de porteurs dans des couches d'épaisseur de deux ordres de grandeur plus petite que les plaquettes de silicium cristallin, c'est-à-dire de l'ordre du $\mu \mathrm{m}$. Ceci est un facteur essentiel du photovoltaïque basé sur des couches minces de a-Si:H.
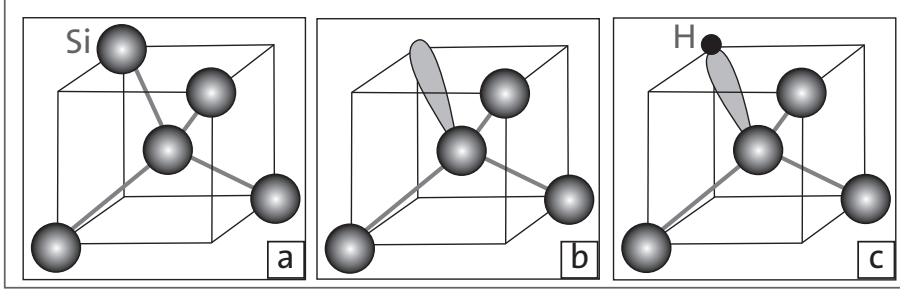

Figure A

a : silicium tétracoordonné

$b$ : silicium tricoordonné avec une liaison pendante.

$c$ : liaison pendante saturée par un atome d'hydrogène.

\section{Références}

[1] W.E. Spear et P.G. Le Comber, Solid State Commun. 17 (1975)

1193.

[2] R.A. Street dans Hydrogenated Amorphous Silicon, Cambridge University Press (1991).

[3] P. Roca i Cabarrocas,

Th. Nguyen-Tran, Y. Djeridane,

A. Abramov, E. Johnson

et G. Patriarche, J. Phys. D: Appl.

Phys. 40 (2007) 2258-2266.

[4] P.W. Anderson, Phys. Rev. 109 (1958) 1492.

[5] N.F. Mott et E.A. Davis,

Electronic Processes in Non-

Crystalline Materials, 2nd Ed.,

Clarendon Press, Oxford (1979).

[6] S. Miyajima, A. Yamada

et M. Konagai, Jpn. J. Appl. Phys.

43 (2004) L1190.

[7] A. Ricaud dans Techniques de l'Ingénieur: Modules

photovoltaïques. Filières

technologiques. D 3-940. 